\title{
THE
}

\section{Turbulent Airflow at Young Sea States with Frequent Wave Breaking Events: Large-Eddy Simulation}

\author{
Nobuhiro Suzuki \\ University of Rhode Island \\ Tetsu Hara \\ University of Rhode Island, thara@uri.edu \\ Peter P. Sullivan
}

Follow this and additional works at: https://digitalcommons.uri.edu/gsofacpubs

\section{Citation/Publisher Attribution}

Suzuki, N., Hara, T., \& Sullivan, P. P. (2011). Turbulent Airflow at Young Sea States with Frequent Wave Breaking Events: Large-Eddy Simulation. Journal of the Atmospheric Sciences, 68, 1290-1305. doi: 10.1175/2011JAS3619.1

Available at: http://dx.doi.org/10.1175/2011JAS3619.1

This Article is brought to you for free and open access by the Graduate School of Oceanography at DigitalCommons@URI. It has been accepted for inclusion in Graduate School of Oceanography Faculty Publications by an authorized administrator of DigitalCommons@URI. For more information, please contact digitalcommons-group@uri.edu. 


\title{
Turbulent Airflow at Young Sea States with Frequent Wave Breaking Events: Large-Eddy Simulation
}

\author{
NOBUhiro SuZUKi AND TETSu HARA \\ Graduate School of Oceanography, University of Rhode Island, Narragansett, Rhode Island \\ Peter P. Sullivan \\ National Center for Atmospheric Research,* Boulder, Colorado
}

(Manuscript received 30 July 2010, in final form 30 December 2010)

\begin{abstract}
A neutrally stratified turbulent airflow over a very young sea surface at a high-wind condition was investigated using large-eddy simulations. In such a state, the dominant drag at the sea surface occurs over breaking waves, and the relationship between the dominant drag and local instantaneous surface wind is highly stochastic and anisotropic. To model such a relationship, a bottom boundary stress parameterization was proposed for the very young sea surface resolving individual breakers. This parameterization was compared to the commonly used parameterization for isotropic surfaces. Over both the young sea and isotropic surfaces, the main near-surface turbulence structure was wall-attached, large-scale, quasi-streamwise vortices. Over the young sea surface, these vortices were more intense, and the near-surface mean velocity gradient was smaller. This is because the isotropic surface weakens the swirling motions of the vortices by spanwise drag. In contrast, the young sea surface exerts little spanwise drag and develops more intense vortices, resulting in greater turbulence and mixing. The vigorous turbulence decreases the mean velocity gradient in the roughness sublayer below the logarithmic layer. Thus, the enhancement of the air-sea momentum flux (drag coefficient) due to breaking waves is caused not only by the streamwise form drag over individual breakers but also by the enhanced vortices. Furthermore, contrary to an assumption used in existing wave boundary layer models, the wave effect may extend as high as 10-20 times the breaking wave height.
\end{abstract}

\section{Introduction}

The atmosphere and ocean are coupled via the fluxes of momentum, heat, and gas at the air-sea boundary. These fluxes are intimately related to the near-surface airflow turbulence. This turbulence is affected in turn by the geometry and motion of water surface gravity waves.

The influence of the waves divides the atmospheric surface layer in two sublayers: the wave boundary layer (WBL) and the inertial sublayer. The WBL is the region contiguous to the water surface and is directly affected by the waves (Sjöblom and Smedman 2003; Sullivan and

\footnotetext{
* The National Center for Atmospheric Research is sponsored by the National Science Foundation.
}

Corresponding author address: Nobuhiro Suzuki, Graduate School of Oceanography, University of Rhode Island, 215 South Ferry Rd., Narragansett, RI 02882.

E-mail: nsuzuki@gso.uri.edu
McWilliams 2010). It is a type of roughness sublayer: the region over a rough wall directly affected by the roughness elements. The inertial sublayer is the region above the WBL, and its mean wind profile approaches the Monin-Obukhov similarity profile. The flow in this layer is indirectly affected by the rough surface as the WBL forms the lower boundary conditions for the inertial sublayer.

Properties of the WBL change with the relative relationship between wind and waves. A wave-wind state where most of the waves are propagating at speeds much slower than the mean near-surface wind is called a young sea state. In such a state, the energy of the airflow, on average, transfers to the wave motions through the work done by the boundary surface forces. This energy input causes the waves to break frequently. In addition, breaking wave crests are sharp and induce airflow separation (Veron et al. 2007), which leads to a large pressure difference between the windward and leeward sides of each breaking crest. Owing to this large pressure 
difference, the form drag over breaking waves becomes very large compared to the other drag forces (i.e., the form drag over nonbreaking waves and the viscous drag at the water surface). Therefore, breaking waves become dominant roughness elements of young sea surfaces (Kukulka and Hara 2008a).

Because of this association with breaking waves, the dominant horizontal drag in young sea states is stochastic and anisotropic. The stochastic drag-wind relationship (Wyngaard et al. 1998) is due to the breaking waves occurring intermittently and independently of the local wind. The anisotropy of the dominant drag originates from the anisotropy of the breaking crest shapes and orientations. In the asymptotic limit of very young sea states, the orientations of breaking crests have little directional spreading, being nearly perpendicular to the near-surface mean wind direction (e.g., Kukulka and Hara 2008b). This implies that their shapes have exceedingly longer along-crest lengths than crosscrest lengths. As pressure force acts only normal to the surface, the geometry of a breaking wave dictates the direction of the dominant drag acting on a local instantaneous wind attacking the breaking wave; the local dominant drag is aligned with the mean wind, not with the local wind. If we call the near-surface mean wind direction the streamwise direction and the horizontal direction perpendicular to the streamwise direction the spanwise direction, then the spanwise component of the local attacking wind receives little opposition by the dominant drag.

The directionality of the dominant drag makes very young sea surfaces akin to $k$-type $2 \mathrm{D}$ transverse roughness and unique compared to smooth walls and $3 \mathrm{D}$ roughness. Here, $k$-type 2D transverse roughness refers to a type of roughness similar to bars mounted spanwise with large distances between the bars, and 3D roughness refers to such roughness that has no preferred directionality (e.g., sand surfaces). Unlike the very young sea surfaces, the dominant drag (regardless of viscous drag or form drag) over smooth walls or 3D roughness is horizontally isotropic; that is, both streamwise and spanwise components of local attacking wind are opposed by the local dominant drag. This difference in the boundaryforcing directionality may cause difference in the airflow turbulence over the anisotropic very young sea surfaces from that over the isotropic smooth or 3D rough surfaces.

There is a substantial literature on the turbulence over water surfaces when the wind is low to moderate (e.g., Sullivan et al. 2000, 2008). In contrast, knowledge of turbulence over water surfaces is limited when the wind is strong. High-wind conditions are of great interest since they occur during important natural events such as tropical cyclones. Thus, a goal of this paper is to develop a deeper understanding of the airflow turbulence over water surfaces at high-wind conditions in neutral stratification. In particular, we will focus on a very young sea state - an idealized extreme case - to highlight the physics involved.

\section{Background on wall-bounded turbulence structures and horizontally anisotropic boundary forcing}

In this section, we briefly review previous studies on coherent structures of neutrally stratified, wall-bounded turbulence and their response to an anisotropic horizontal drag, since they are relevant to this study.

There is growing evidence that large coherent turbulence structures over smooth walls and rough walls are qualitatively similar (Flack et al. 2007; Morris et al. 2007; Lee et al. 2009; Volino et al. 2009). These structures are large quasi-streamwise vortices (QSVs) extending from the logarithmic layer and attached to the wall. Over smooth walls, these large QSVs are shown to be induced by the tall wall-attached vortex clusters or packets (Tomkins and Adrian 2003, 2005; del Álamo et al. 2006). These packets and QSVs exist in many different sizes. They are important near the wall because they carry significant turbulent kinetic energy and Reynolds stress (Balakumar and Adrian 2007; Tomkins and Adrian 2005).

Unlike the qualitative similarity in the large-scale QSVs over smooth and rough walls, small-scale QSVs are greatly disrupted by the roughness when their heights are comparable or less than the roughness elements. They are shortened and disoriented because of the collision with the roughness elements (Ikeda and Durbin 2007). Thus, the effect of roughness on a QSV seems distinctly different depending on the height of the QSV relative to the height of the roughness element. For a relatively tall QSV, roughness exerts drag on the nearwall side of the vortex. In contrast, for a relatively short QSV, roughness impedes the ordinary advection of the vortex as if the roughness element were an obstacle in its path.

Wall-attached streamwise vortices are closely related to the mean velocity gradient. The mean velocity gradient likely stimulates production of the wall-attached QSVs (del Álamo et al. 2006). In turn, the QSVs have negative feedback on the mean velocity gradient because they cause vertical mixing. If the Reynolds shear stress is held constant, then the mean velocity gradient decreases (increases) when the streamwise vortices are enhanced (hindered). This relationship has been observed using many methods that affect the intensity of the small-scale, wall-attached streamwise vortices. Note 
that much knowledge of QSV physics has been derived from the small-scale streamwise vortices, possibly because the large-scale QSVs are difficult to identify (Morris et al. 2007) and difficult to simulate using small domain sizes of direct numerical simulations. Examples of these methods are wall-mounted streamwise riblets (Choi et al. 1993), active blowing at the wall (Choi et al. 1994), wall oscillation (Karniadakis and Choi 2003), and addition of polymer to the fluid (Kim et al. 2008).

Another example of these methods is anisotropic slip conditions over flat walls (Min and Kim 2004). Such boundary conditions can be realized by a streamwise noslip condition used together with a spanwise slip condition, and vice versa. In this method, an increase of the spanwise slip enhances the wall-attached, streamwise vortices and thereby decreases the mean velocity gradient off but near the wall. The intensity of the streamwise vortices is controlled this way since the spanwise friction at the wall acts on and dissipates the swirling motions of the streamwise vortices.

Little is known about the relationship between the relatively large wall-attached QSVs and the anisotropic boundary forcing due to roughness. However, it may be possible that the relationship is essentially the same as the foregoing relationship between the small-scale streamwise vortices and the anisotropic friction. Since $k$-type transverse roughness (including very young sea surfaces) exerts little spanwise form drag, the large wallattached QSVs would be enhanced. In contrast, since 3D roughness exerts large spanwise form drag, the large wall-attached QSVs would be hindered. As the large wall-attached QSVs are very energetic, this difference could be important. Indirect evidence might be seen in the experiment by Volino et al. (2009) where $k$-type 2D transverse roughness is compared to $3 \mathrm{D}$ roughness in the fully rough regime. They reported that, over the transverse roughness, the ratio of the equivalent sand roughness (i.e., about 30 times the roughness length) to the actual physical height of the roughness elements is much larger and the effect of roughness extends farther away from the wall. Therefore, their results imply that if we compare a $k$-type $2 \mathrm{D}$ transverse roughness and a $3 \mathrm{D}$ roughness having the same roughness-element height and mean drag, the transverse roughness would yield smaller overall mean velocity gradient near the wall. This is consistent with our expectation that more intense QSVs are possible over the transverse roughness.

Based on these previous studies, we anticipate that the large-scale, wall-attached QSVs are the dominant features of turbulence over sea surfaces. The QSVs may be enhanced and the mean velocity gradient in the WBL may be reduced over young seas because of the directionality of the boundary forcing. We will therefore pay particular attention to QSVs and their responses to different bottom boundary conditions in this study.

\section{Methods}

\section{a. Outline}

The very-high-Reynolds-number airflows under consideration are simulated using large-eddy simulation (LES). To include breaking waves in the simulations, our LES take a simplified approach similar to the typical LES studies of airflow turbulence over canopies (e.g., Dwyer et al. 1997). That is to say, instead of explicitly simulating the local form drag resulting from the interaction between airflow and a breaking wave, the bottom boundary is made flat, and the form drag is diagnosed using a parameterization.

A time-dependent spatial distribution of breaking crests is generated independently of the local LES winds. At each time step and position, our parameterization diagnoses an external force representing the local form drag based on the local LES wind and the local breaking crests. In this study we set the LES vertical grid spacing such that no breaking waves are taller than the LES grid boxes adjacent to the bottom boundary. Thus, the form drag explicitly appears only at the lowest grid level and is included in the bottom boundary stress parameterization.

In the following, we will mainly consider three idealized bottom boundary stress parameterizations in order to highlight the impact of the intermittent and anisotropic horizontal form drag. They are a 3D roughness model; a highly anisotropic, intermittent, very young sea surface model; and a highly anisotropic, very young sea surface model without the intermittency (i.e., uniform occurrence of the breaking crests). The first parameterization is proposed by Grötzbach (1987) and has been widely used to simulate wall-bounded, high-Reynoldsnumber turbulent flows (e.g., Stoll and Porté-Agel 2006; Senocak et al. 2007). The latter two parameterizations are newly proposed in this paper. Comparison between the latter two models highlights the effect of anisotropy separately from the intermittency. In addition to these idealized young sea surfaces, we will briefly consider more realistic young sea surfaces.

\section{b. Governing equations}

Let $(x, y, z)$ be a Cartesian coordinate system, where $x$ and $y$ are in the horizontal directions and $z$ is in the vertical direction measured upward from the bottom surface located at $z=0$. Let $(u, v, w)$ be the components of the filtered (or resolved) velocity $\mathbf{u}$ in the coordinate directions $(x, y, z)$. 
The flow is assumed to be incompressible. Then the LES equations for $\mathbf{u}$ and filtered virtual potential temperature $\theta$ are

$$
\begin{aligned}
\nabla \cdot \mathbf{u} & =0, \\
\frac{\partial \mathbf{u}}{\partial t} & =\mathbf{u} \times \nabla \times \mathbf{u}-\nabla \Pi+\Delta-\nabla \cdot \boldsymbol{\tau}+\frac{\mathbf{g}}{\theta_{0}}\left(\theta-\theta_{0}\right), \\
\frac{\partial \theta}{\partial t} & =-\nabla \cdot(\theta \mathbf{u})-\nabla \cdot \boldsymbol{\tau}_{\theta},
\end{aligned}
$$

where $\Pi=p^{\prime} / \rho_{0}+(2 / 3) e_{\mathrm{SGS}}+(1 / 2) \mathbf{u} \cdot \mathbf{u}$ is the generalized pressure; $p^{\prime}$ is the deviation of the filtered pressure from its horizontal mean; $\Delta=(\Delta, 0,0)$ is the externally imposed, constant, mean pressure gradient force; $e_{\mathrm{SGS}}$ is the subgrid-scale (SGS) turbulent kinetic energy (TKE); $\rho_{0}$ is the reference density of air; $\mathbf{g}=(0,0, g)$ is the gravitational acceleration; $\theta_{0}$ is the reference potential temperature; and $\boldsymbol{\tau}$ and $\boldsymbol{\tau}_{\theta}$ are the SGS flux terms. Note that the definition of $\tau$ follows the LES convention and its sign is different from that of the standard turbulent Reynolds stress.

There are various models of the SGS flux terms. Different SGS models yield very different airflow turbulence near a smooth wall or 3D roughness (e.g., Brasseur and Wei 2010). To distinguish the effects of the bottom boundary stress models from the biases associated with SGS models, most simulations are repeated with two commonly used SGS models. One is the SGS TKEbased eddy viscosity SGS model used by Moeng (1984), and the other is the two-part SGS model developed by Sullivan et al. (1994).

Although all our simulations are performed for a neutral stratification, Eq. (3) is used to apply a small, surface heat flux for a short time at the beginning of the simulations. This allows a quick spinup of the turbulence and smooth convergence to statistically steady, neutrally stratified shear turbulent flow (Moeng and Sullivan 1994).

In this study, the Coriolis force is ignored, and the flow is driven by the externally imposed mean pressure gradient in the positive $x$ direction. Thus, in an equilibrium state, the resultant mean wind is in the positive $x$ direction. The resultant vertical profile of the mean stress is linear (in contrast to the constant stress profile) and the mean pressure gradient force is balanced by the vertical gradient of the mean stress. The $x$ direction is the streamwise direction, and the $y$ direction is the spanwise direction. The value of $\Delta$ is chosen in such a way that the mean surface friction velocity $U_{*}$ is $2 \mathrm{~m} \mathrm{~s}^{-1}$ in an equilibrium state.

\section{c. Model of breaking-wave distribution}

The breaking crest field generated for the young sea surface parameterizations consists of breaking waves with a wide range of wavenumbers. Each of these breaking crests has its own length, propagation speed, and lifetime. Initiation of individual breaking crests occurs intermittently in time and space in such a way that the resultant breaking crest field satisfies, on average, a particular "breaking-wave distribution" $\Lambda(k, \sigma)$ at each wavenumber $k$ and orientation angle $\sigma$. Here, the breaking-wave distribution refers to such a function that $\Lambda(k, \sigma) d k d \sigma$ represents the average length of breaking crests per unit horizontal surface area for waves with wavenumbers between $k-d k / 2$ and $k+d k / 2$ and angles between $\sigma-d \sigma / 2$ and $\sigma+d \sigma / 2$ (Phillips 1985).

In this study, $\Lambda$ is specified based on the theoretical models by Kukulka and Hara (2008a,b) and Kukulka et al. (2007). The former model is based on the conservation of wave energy and the conservation of both airside momentum and energy for a fully developed airflow turbulence over very young to mature seas. In their models, variables are normalized in such a way that the results depend only on the wave age $c_{p} / U_{*}$, where $c_{p}$ is the phase speed at the spectral peak. The predicted $\Lambda$ is consistent with existing observations in open ocean conditions, where seas are more developed and the wave age is about 10 or larger. In younger sea states, direct observations of $\Lambda$ are not readily available. However, there are indirect validations against laboratory experiments conducted at wind speed of 30 and $50 \mathrm{~m} \mathrm{~s}^{-1}$, in which the model predictions of the drag coefficient are consistent with the observed values. In very young sea states, the model predicts that the majority of the wind stress is supported by the form drag over breaking waves (i.e., the form drag over nonbreaking waves and the surface viscous stress become negligibly small). The model also predicts that the directional spreading of $\Lambda$ becomes narrower for younger seas, and it becomes unidirectional in the asymptotic limit of very young sea states. Finally, the model is well approximated by the simpler model of Kukulka et al. (2007) for wave ages less than one. Thus, we set the wave age to 0.92 and simply use $\Lambda(k)$ by Kukulka et al. (2007). (Our future study will address simulations with older seas.)

In addition to a solution of $\Lambda$, the model of Kukulka et al. (2007) predicts a vertical profile of the mean wind velocity $\langle u\rangle$ below the height of the tallest breaking wave as well as a solution of the mean form $\operatorname{drag} s\left(k_{1}, k_{2}\right)$ supported by the breaking waves of wavenumbers between $k_{1}$ and $k_{2}$ (where $k_{1}<k_{2}$ ). Here, $\langle\cdot\rangle$ denotes horizontal and long time averaging. Their model requires specification of $U_{*}$ and smallest wavenumber $k_{0}$ of the 
young sea condition to be considered. Then their solutions are given by

$$
\begin{aligned}
\Lambda(k) & =\frac{\rho_{a}}{b \rho_{w}}\left(\frac{u_{\ell^{*}}}{c}\right)^{2}\left(\frac{\gamma}{\kappa} \frac{u_{\ell^{*}}}{c}-\frac{1+\gamma}{2}\right), \\
\langle u\rangle & =\left(1+\gamma^{-1}\right) \sqrt{g z / \epsilon} \quad\left(\text { for } z \leq \epsilon / k_{0}\right), \\
s\left(k_{1}, k_{2}\right) & =\tau_{t}\left(k_{1}\right)-\tau_{t}\left(k_{2}\right)=\rho_{a}\left[u_{\ell^{*}}^{2}\left(k_{1}\right)-u_{\ell^{*}}^{2}\left(k_{2}\right)\right],
\end{aligned}
$$

with

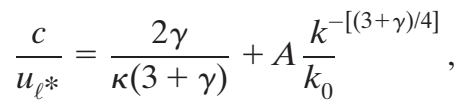

where $c(k)$ is the phase speed that is approximated by the linear deep water dispersion relation, $u_{\ell^{*}}(k)$ is $\sqrt{\tau_{t}(k) / \rho_{a}}, \tau_{t}(k)$ is the Reynolds averaged turbulent wind stress at $z=\epsilon / k, \rho_{a}$ is the density of air, $\rho_{w}$ is the density of water, $\epsilon=0.3$ is the assumed slope of the breaking waves, $\kappa=0.4$ is the von Kármán constant, $\gamma$ is $\sqrt{2 c_{d} \epsilon \rho_{a} /\left(b \rho_{w}\right)}, c_{d}$ is the form drag coefficient of a breaking wave, $b$ is the energy dissipation coefficient of breaking waves, and $A$ is a constant determined such that $u_{\ell^{*}}\left(k_{0}\right)=U_{*}$.

In our study, the young sea condition of wave age 0.92 is specified by $k_{0}=2.88 \mathrm{rad} \mathrm{m}^{-1}$ and $U_{*}=2 \mathrm{~m} \mathrm{~s}^{-1}$ since the LES code is written for dimensional variables and we need to specify the dominant wave scale and wind forcing. However, our LES results are in principle applicable at different friction velocities provided the wave age is 0.92 . The two model parameters $b$ and $\gamma$ are set at $b=0.01$ and $\gamma=0.5$ following Kukulka et al. (2007).

These breaking-crest statistics, Eqs. (4) and (5), are held constant during the LES since the time required for the airflow turbulence to fully develop is much shorter than the time scale of the wave-field evolution. It is worth mentioning that although we chose this particular $\Lambda$, our results turned out to be relatively insensitive to particular forms of $\Lambda$.

\section{d. Instantaneous breaking crest field}

To generate an intermittent, time-dependent, breaking crest field that satisfies the above statistical constraints, we need to also specify the size, time, and location of occurrence, lifetime, and propagation speed of every individual breaking wave. The length of an individual breaking crest is set equal to the wavelength $\lambda=2 \pi / k$ of the corresponding wave, and its lifetime is set to one wave period $T=2 \pi / \omega$ based on the laboratory observations by Melville et al. (2002). Here, the angular frequency $\omega$ is related to the wavenumber through the linear deep water dispersion relation $\omega^{2} \equiv(c k)^{2}=g k$.

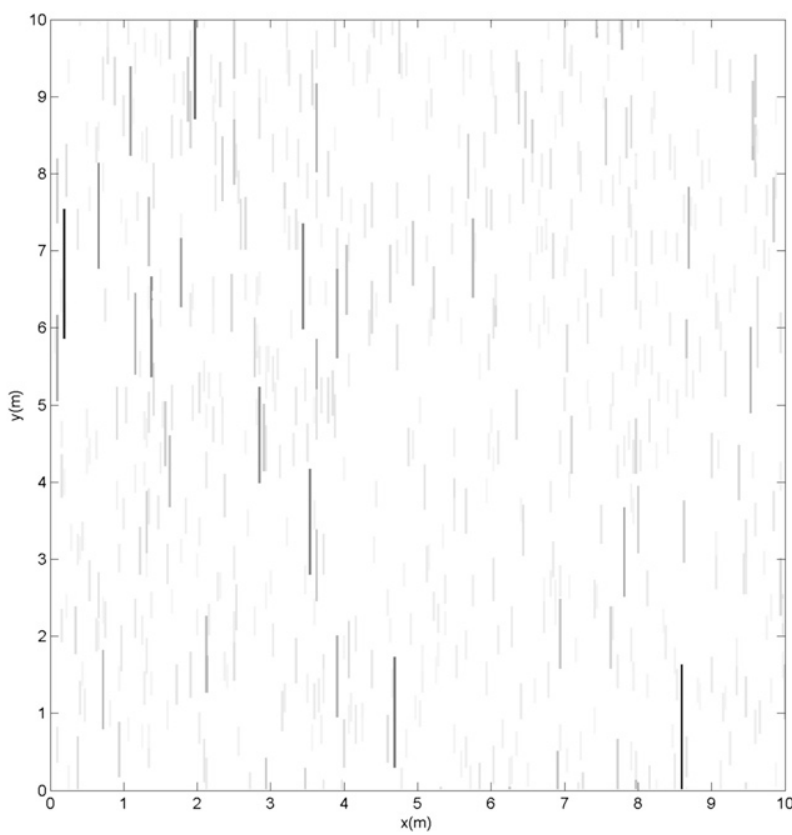

FIG. 1. An example of instantaneous breaking wave crest positions. The darker the line shading, the smaller the wavenumber associated with it.

The propagation speed of the individual breaking wave is set to $[c(k), 0,0]$ following Kukulka et al. (2007). We have set these parameters following Sullivan et al. (2004), who used similar scales for these quantities. Again, our results turned out to be relatively insensitive to the values of these parameters.

Independent of the airflow above, breaking events are initiated intermittently and randomly in space and time. A random number of breaking crests at each $k$ is initiated at each time step in such a way that the resultant breaking wave field satisfies Eq. (4) on a long time average over the entire bottom boundary. Once generated, each breaking wave moves at its own propagation speed and lives for its own lifetime.

A snapshot of the breaking wave crest field generated is shown in Fig. 1.

\section{e. Bottom boundary stress models}

\section{1) 3D ROUGHNESS MODEL}

The vertical flux of streamwise momentum $\tau_{x z}$ and that of spanwise momentum $\tau_{y z}$ at $3 \mathrm{D}$ rough surfaces are commonly modeled as being aligned and opposite to the local attacking wind (e.g., Piomelli 2008; Pope 2000):

$$
\begin{aligned}
& \tau_{x z}(x, y, 0, t)=-\frac{\overline{\tau_{w}}(t)}{\bar{u}\left(z_{1}, t\right)} u\left(x, y, z_{1}, t\right), \\
& \tau_{y z}(x, y, 0, t)=-\frac{\overline{\tau_{w}}(t)}{\bar{u}\left(z_{1}, t\right)} v\left(x, y, z_{1}, t\right),
\end{aligned}
$$


where $z_{1}$ is the first off-surface grid level (i.e., the first $u$-grid level for our staggered grid), $\tau_{w}$ is the bottom boundary stress, and the overbar denotes the horizontal spatial average.

This parameterization is often used together with another parameterization for the mean bottom boundary stress $\overline{\tau_{w}}$. If there is (approximately) a constant stress layer near the bottom boundary and $z_{1}$ is located within it, then $\overline{\tau_{w}}(t)$ is computed at each time step using the Monin-Obukhov theory (used instantaneously) in neutrally stable conditions:

$$
\overline{\tau_{w}}(t)=\left[\frac{\kappa}{\log \left(z_{1} / z_{0}\right)}\right]^{2}\left|\bar{u}\left(z_{1}, t\right)\right| \bar{u}\left(z_{1}, t\right)
$$

where $z_{0}$ is the roughness length of the specified surface. Since $\left|\left\langle\tau_{w}\right\rangle\right| \equiv U_{*}^{2}$ in the equilibrium state, the input value of $z_{0}$ controls the value of $\langle u\rangle$ at $z_{1}$ in the equilibrium state.

In this study, we will compare 3D and young sea surfaces having the same mean surface momentum flux and the same mean velocity at $z_{1}$ in their equilibrium states. Based on Eq. (5), this mean velocity is chosen to be $\left(1+\gamma^{-1}\right) \sqrt{g z_{1} / \epsilon}$, with $z_{1}$ being at or below $\varepsilon / k_{0}$. Then, Eq. (10) in the equilibrium state defines the value of $z_{0}$; that is,

$$
U_{*}=\frac{\kappa}{\log \left(z_{1} / z_{0}\right)}\left(1+\gamma^{-1}\right) \sqrt{g z_{1} / \epsilon} .
$$

\section{2) Highly ANISOTROPIC, INTERMitTENT, YOUNG SEA SURFACE MODEL}

The form-drag parameterization here follows that of Kukulka et al. (2007) except for the following two modifications:
1) In Kukulka et al. (2007), the form drag over a particular breaking wave is proportional to its frontal area (the height times the length of the breaking wave crest) times the square of the relative mean wind speed attacking its crest. In this study, the LES calculate the wind speed at $z_{1}$ but do not resolve the wind profile below. Therefore, the form drag is parameterized in terms of the difference between the local wind speed at $z_{1}$ and the breaking crest propagation speed.

2) In Kukulka et al. (2007), the form drag due to a breaking wave is concentrated along its crest (line). In this study, however, the form drag is distributed over the horizontal area spanned by the breaking wave (breaking crest length times wavelength). This modification is needed because the local boundary stress due to a large breaking wave (relative to the LES grid size) becomes unrealistically large if the form drag is concentrated on its line crest.

In this study, breaking waves are allowed to overlap, and every breaking wave at a point on the water surface is assumed to support form drag. Thus, the local net form drag is found by summing all the form drag over the breaking waves present at that point. Breaking waves that are much smaller than the LES grid size occur so frequently that their contribution to the form drag is practically uniform in both space and time on the LES grid. Therefore, the breakers whose wavenumbers are greater than a cutoff wavenumber $k_{t}$ do not need to be explicitly resolved. Only the breakers whose wavenumbers are less than $k_{t}$ are explicitly generated in the input breaking crest field in order to account for their intermittency on the LES grid. Specifically, the highly anisotropic, intermittent, young sea model is expressed as

$$
\tau_{x z}(x, y, 0, t)=-\int_{k_{0}}^{k_{t}} \sum_{m=1}^{N(k, x, y, t)} C_{D}(k)\left|\bar{u}\left(z_{1}, t\right)-c(k)\right|\left[u\left(X_{m}, y, z_{1}, t\right)-c(k)\right] d k-\left|\frac{s\left(k_{t}, \infty\right)}{\left.\rho_{a}\langle u\rangle\right|_{z=z_{1}}}\right| u\left(x, y, z_{1}, t\right),
$$

$$
\tau_{y z}(x, y, 0, t)=0
$$

where $N(k, x, y, t)$ is the total number of breaking waves of wavenumber between $k-d k / 2$ and $k+d k / 2$ present at $(x, y, t), X_{m}$ is the $x$ coordinate of the crest of the $m$ th breaking wave where the wind attacks the breaking wave, $C_{D}(k)$ is an empirical drag coefficient (per unit wavenumber), and $\left.\langle u\rangle\right|_{z=z_{1}}$ is the mean velocity at $z=z_{1}$ obtained by Eq. (5). When $N(k, x, y, t)=0$, there is no term to be summed for that $k$. The value of $C_{D}(k)$ is defined such that, in the equilibrium state, the average of the simulated form drag at each $k$ is the same as that of the constraining statistics Eq. (6); that is, at each $k$ less than $k_{t}$,

$$
\begin{aligned}
& \left\langle\sum_{m=1}^{N(k, x, y, t)} C_{D}(k)\left|\bar{u}\left(z_{1}, t\right)-c(k)\right|\left[u\left(X_{m}, y, z_{1}, t\right)-c(k)\right] d k\right\rangle \\
& =\frac{s(k-d k / 2, k+d k / 2)}{\rho_{a}}
\end{aligned}
$$

The first term in Eq. (12) models the form drag due to the explicitly generated (i.e., resolved) breaking waves. 


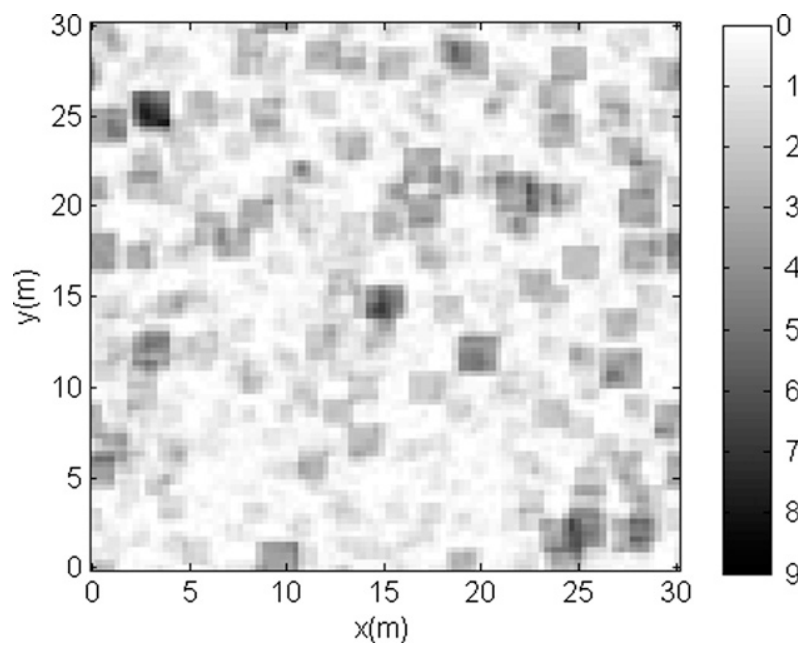

FIG. 2. An example of breaking wave field. The shading shows $\int_{k_{0}}^{k_{t}} \sum_{m=1}^{N(k, x, y, t)} C_{D}(k) d k$ in Eq. (12).

The second term models the form drag due to the unresolved breaking waves, whose wavenumber is larger than $k_{t}$. For this model, the cutoff wavenumber is set at $k_{t}=20.9 \mathrm{rad} \mathrm{m}^{-1}$. The results are not affected when the value of $k_{t}$ is increased. About $72 \%$ of the total drag is supported by the intermittent resolved breakers at this $k_{t}$, and the rest is by the unresolved breakers.

We are aware that some variations of Eq. (12) are possible. For example, $\left|u\left(X_{m}, y, z_{1}, t\right)-c\right|\left[u\left(X_{m}, y, z_{1}, t\right)-c\right]$ may be used instead of $\left|\bar{u}\left(z_{1}, t\right)-c\right|\left[u\left(X_{m}, y, z_{1}, t\right)-c\right]$. In this case, the value of $C_{D}(k)$ must be adjusted iteratively in order to satisfy its counterpart of Eq. (14). We compared these two variations and our results did not show significant differences.

Figure 2 shows a snapshot of the spatially distributed drag coefficients.

\section{3) Highly ANISOTROPIC, NONINTERMITTENT, YOUNG SEA SURFACE MODEL}

When all breaking waves are treated as unresolved (i.e., they occur uniformly in time and space with respect to the LES temporal and spatial resolution), Eqs. (12) and (13) reduce to

$$
\begin{aligned}
& \tau_{x z}(x, y, t)=-\left|\frac{U_{*}^{2}}{\left.\langle u\rangle\right|_{z=z_{1}}}\right| u\left(x, y, z_{1}, t\right), \\
& \tau_{y z}(x, y, t)=0 .
\end{aligned}
$$

Note that $\tau_{x z}$ is effectively the same as that of the 3D roughness model Eq. (8). The only remaining difference is the anisotropy, Eq. (16). Even if all the breaking waves are distributed uniformly, the momentum transfer from wind to breaking waves are still via the pressure form drag acting normal to the breaking waves.

\section{f. Numerical method}

The pseudospectral method for horizontal derivatives and the second-order centered finite difference scheme for vertical derivatives are used to discretize Eqs. (1)-(3) on a vertically staggered grid. The variables $u, v, \theta$, and $\Pi$ are stored at the $u$-grid levels, and $w$ and $e_{\mathrm{SGS}}$ are stored at the $w$-grid levels. The bottom and upper boundaries are located at $w$-grid levels, and the $u$-grid levels are located midway between the neighboring $w$-grid levels.

Time integration is made using an explicit, thirdorder, three-substep Runge-Kutta scheme. The time step is held fixed at $\Delta t=0.01$ or $0.004 \mathrm{~s}$ depending on the spatial resolution. These time steps are smaller than the time steps based on a fixed Courant-Fredrichs-Lewy (CFL) condition. The pressure is determined to ensure the incompressibility at the end of each substep. The boundary conditions are horizontally periodic and also nonpermeable and frictionless at the upper boundary. At the bottom surface, $w=0$.

All simulations are made on a uniform $96 \times 96 \times 96$ grid in a $60 \mathrm{~m} \times 60 \mathrm{~m} \times 20 \mathrm{~m}$ domain. The domain height is about 96 times higher than the tallest breaking waves considered. In addition, some simulations are repeated with a higher resolution to investigate the resolution dependence of the solutions. For the higherresolution simulations, a $96 \times 96 \times 96$ grid is used for a $30 \mathrm{~m} \times 30 \mathrm{~m} \times 20 \mathrm{~m}$ domain. In this case, a vertically nonuniform grid is used such that the vertical resolution is about twice as fine near the bottom boundary.

The breaking crest field is simulated without discretization. To compute Eq. (12) at each LES time step, the positions of the breaking waves are mapped onto a very fine $2 \mathrm{D}$ grid covering the LES domain bottom. The local stresses on the 2D-grid nodes located within an LES mesh area are averaged in order to find the local bottom boundary stress on that LES bottom node.

All results are obtained after the flow is converged to a statistically steady (i.e., fully developed) state. The statistics are made by averaging over a horizontal plane and time. To calculate the mean vertical profiles, data are taken every $0.2 \mathrm{~s}$ over $240 \mathrm{~s}$ (i.e., 24 large eddy turnover time defined as domain height divided by $U_{*}$ ). For the quadrant analysis, data are taken every $1 \mathrm{~s}$ over the same time period.

\section{Results}

\section{a. The resolved large-eddy structures}

Figures 3 and 4 show examples of the instantaneous resolved turbulent velocity fluctuations $\mathbf{u}^{\prime}=\mathbf{u}-\overline{\mathbf{u}}$ on 


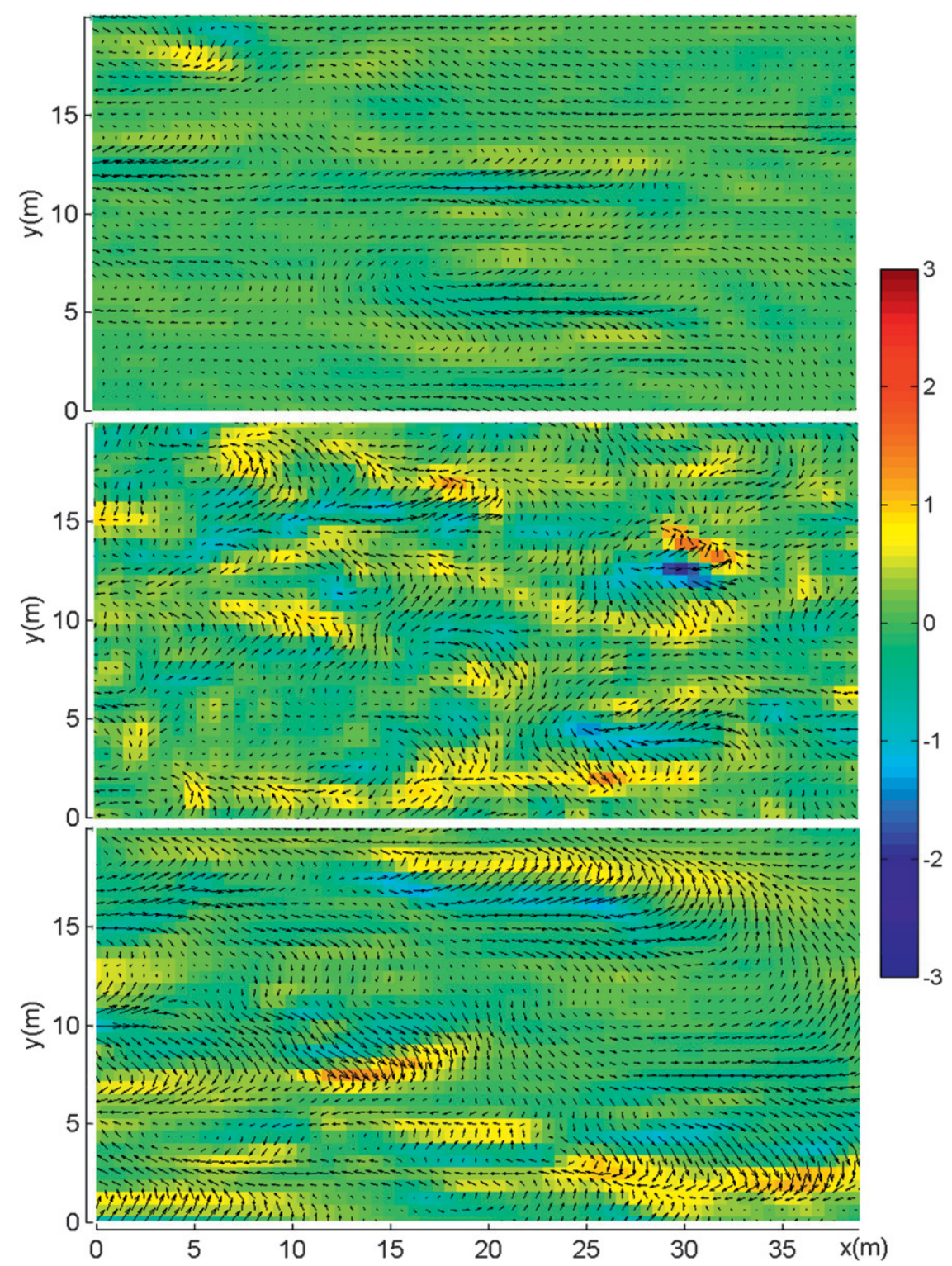

FIG. 3. Instantaneous velocity fluctuations with the SGS TKE-based eddy viscosity SGS model. (top) 3D roughness. (middle) Highly anisotropic intermittent young sea. (bottom) Highly anisotropic nonintermittent young sea. The arrows are $\left(u^{\prime}, v^{\prime}\right)$ at the first $u$ grid $(z=$ $0.1042 \mathrm{~m})$, and the color shows $w^{\prime}$ at the first $w$ grid $(z=0.2083 \mathrm{~m})$ in m s${ }^{-1}$. A horizontal speed of $7 \mathrm{~m} \mathrm{~s}^{-1}$ is shown by a $1-\mathrm{m}$ arrow.

a horizontal plane near the bottom surface. Although each figure shows just one snapshot, it is characteristic of the instantaneous flow field at other times. It is clear that there are much more resolved turbulent fluctuations $u^{\prime}$, $v^{\prime}$, and $w^{\prime}$ above the highly anisotropic very young sea surfaces (intermittent or nonintermittent) compared to the $3 \mathrm{D}$ roughness. In all simulations, $w^{\prime}$ tends to form a pair of positive and negative lines along the quasistreamwise direction. These lines correspond to regions of strong ejections (i.e., negative $u^{\prime}$ associated with positive $w^{\prime}$ ) and sweeps (positive $u^{\prime}$ associated with negative $w^{\prime}$ ). Each pair of positive and negative $w^{\prime}$ motions is continuously connected by a spanwise flow $v^{\prime}$ flowing from the negative $w^{\prime}$ side to the positive $w^{\prime}$ side. This flow pattern is a signature of the lower part of the wall-attached quasi-streamwise vortices. The figures show that the wall-attached QSVs are the dominant resolved-scale turbulence structures at this height, and they are much enhanced over the young sea surfaces compared to the $3 \mathrm{D}$ roughness. 


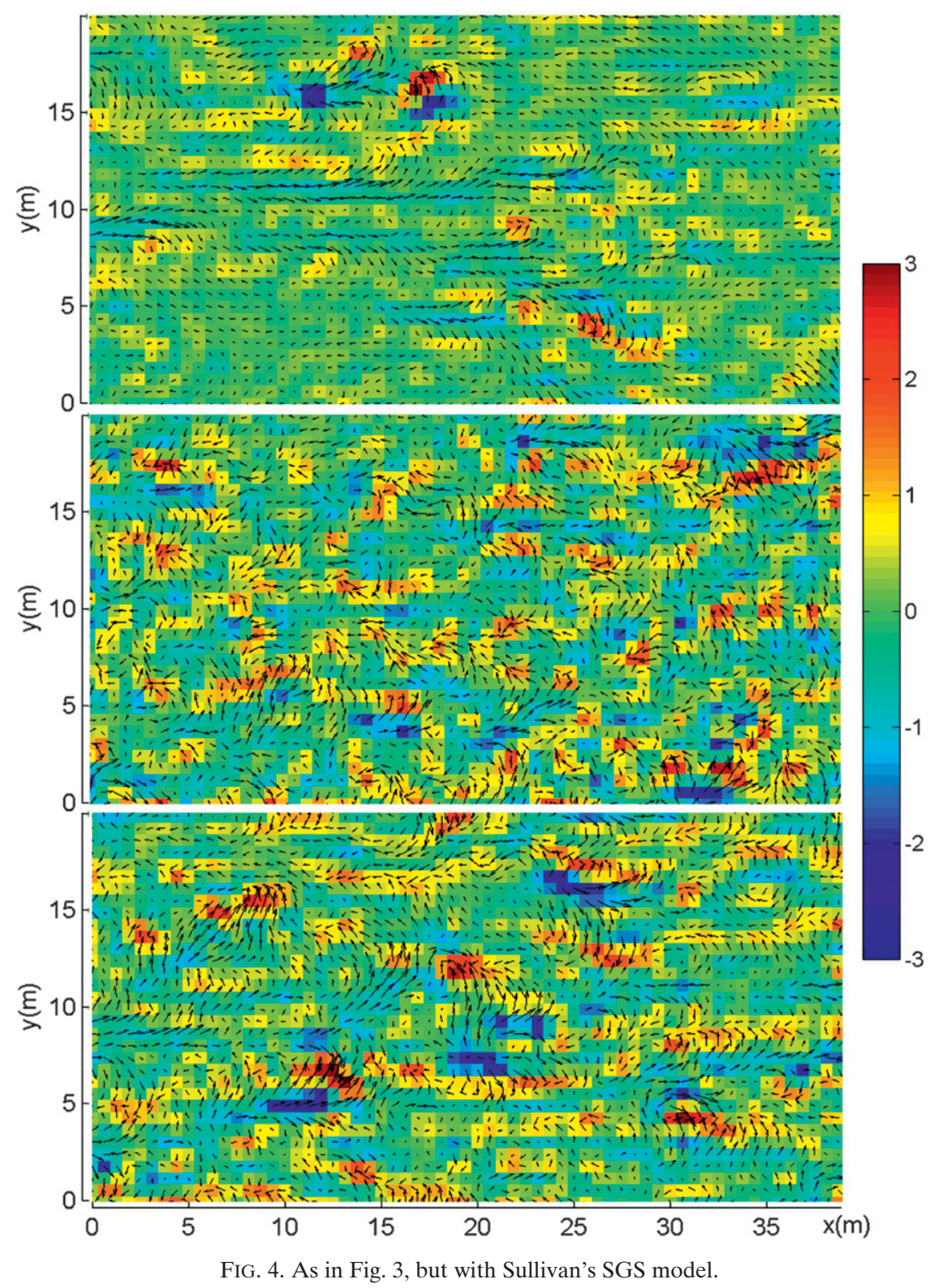

Although it is not as clear, the wall-attached QSVs over the intermittent young sea surface tend to have slightly shorter length compared to the nonintermittent young sea surface. Over the intermittent breaking waves, strong streamwise drag appears abruptly and disrupts the QSVs. This effect of intermittency is weak at the very young sea surface since the heights of the breaking waves are relatively short compared to the discernible QSVs.

Even though the flow fields of the SGS TKE-based eddy viscosity SGS model and those of Sullivan's SGS model are quite different, the effects of young sea surfaces mentioned here appear consistently with both SGS models. These effects are consistent with the expected response of QSVs to the very young sea surfaces as discussed in section 2 .

Figure 5 shows an instantaneous $v^{\prime}$ field on an $x-z$ plane for the 3D roughness model and the highly anisotropic intermittent young sea surface model. The result from the nonintermittent young sea surface model is not shown here since it is very similar to that of the intermittent young sea surface model. The structure of $v^{\prime}$ may be seen as a useful indicator of the QSVs. These large eddies become more vertical farther from the surface, but they are more tilted and stretched by the vertical gradient of $\langle u\rangle$ as they approach the surface. 

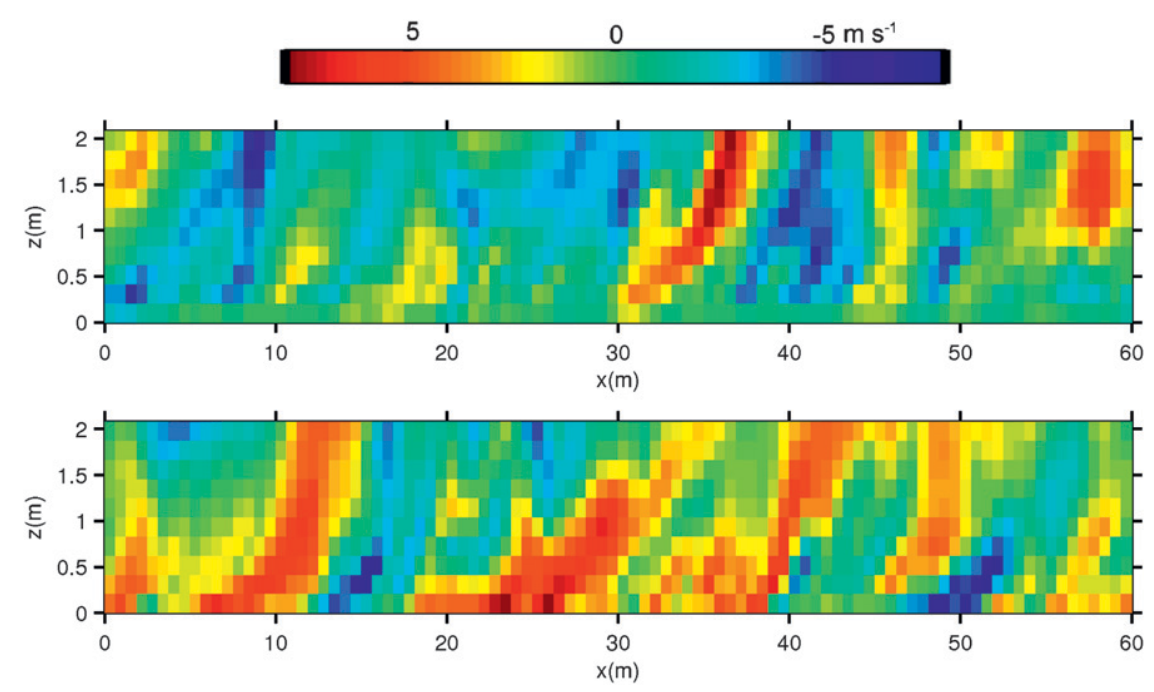

FIG. 5. Instantaneous $v^{\prime}$ field ( $\mathrm{m} \mathrm{s}^{-1}$ ) on an $x-z$ plane. Sullivan's SGS model is used. (top) 3D roughness model; (bottom) highly anisotropic intermittent young sea surface model.

Discernible suppression of $v^{\prime}$ just above the bottom surface is observed over the $3 \mathrm{D}$ roughness model. In contrast, $v^{\prime}$ of the young sea surface models indicates smooth continuation of large vortex structures from the interior to the surface.

\section{b. Statistics of turbulent velocity fluctuations}

Statistics of turbulent velocity fluctuations are shown here in order to quantify the increase of the large-scale coherent turbulence structures seen in the instantaneous flow fields. Figure 6 shows the vertical profiles of the velocity variances, $\left\langle u^{\prime} u^{\prime}\right\rangle,\left\langle v^{\prime} v^{\prime}\right\rangle$, and $\left\langle w^{\prime} w^{\prime}\right\rangle$. Here, the height is normalized by $z_{1}$, which is the height of the tallest breaker as well as the height where the mean wind is matched between the young sea surfaces and the 3D roughness surface. All the velocity variances increase near the bottom surface over the very young sea surfaces compared to the 3D roughness, being consistent with the instantaneous flow fields. Especially, the near-surface $\left\langle v^{\prime} v^{\prime}\right\rangle$ is roughly 4 times larger over the very young sea surfaces. The increase of the other variances ranges between about $20 \%$ and $100 \%$ depending on the SGS models used. The resolved Reynolds shear stress $\left\langle u^{\prime} w^{\prime}\right\rangle$ follows the same trend. It is roughly twice as large over the very young sea surfaces compared to the $3 \mathrm{D}$ roughness (Fig. 7).

Further information about the turbulent momentum flux $u^{\prime} w^{\prime}$ can be obtained from the quadrant analysis. Figure 8 shows an example of the joint probability density function (PDF) of $u^{\prime}$ and $w^{\prime}$ (at the second $u$-grid level obtained with Sullivan's SGS model). Notice that the young sea surface models decrease the weak ejections and sweeps and increase the strong ejections and sweeps, indicating the increase of more coherent structures as we saw in the instantaneous flows. Moreover, the strong ejections are more enhanced than the sweeps. (The results with the other SGS model and at other elevations are qualitatively similar.) This is consistent with Fig. 9 in which $\left\langle\left(w^{\prime}\right)^{3}\right\rangle$ are shown. When strong ejections increase more than the strong sweeps do, $\left\langle\left(w^{\prime}\right)^{3}\right\rangle$ increases.

The differences between the intermittent and nonintermittent young sea surface models are very subtle in these statistics. This again indicates that the effect of intermittency is weak at very young sea conditions, and the effect of the directionality alone causes the significant differences.

In Figs. 6 and 7, the increase of turbulence over the young sea surfaces extends $1-2 \mathrm{~m}$ from the bottom (i.e., 10-20 times higher than the tallest breaking waves), implying that the WBL height is significantly larger than the tallest breaking wave height.

\section{c. Mean velocity profile}

The mean wind profiles and the nondimensional mean gradient profiles $\phi_{m} \equiv z \kappa U_{*}^{-1} d\langle u\rangle / d z$ are shown in Figs. 10 and 11. In both figures, the thin solid lines indicate the profiles of the logarithmic layer; that is, $\langle u\rangle=$ $U_{*} \kappa^{-1} \log \left(z / z_{0}\right)$ and $\phi_{m}=1$. The logarithmic layer is expected to extend up to lower $20 \%(4 \mathrm{~m})$ of the half channel height. Note that for neutrally stratified wall turbulence flows over smooth walls or 3D roughness, most LES do not accurately produce the expected logarithmic profile near the bottom boundary (e.g., Brasseur and Wei 2010). In fact, both SGS models used in this study overpredict the near-surface mean gradient when 

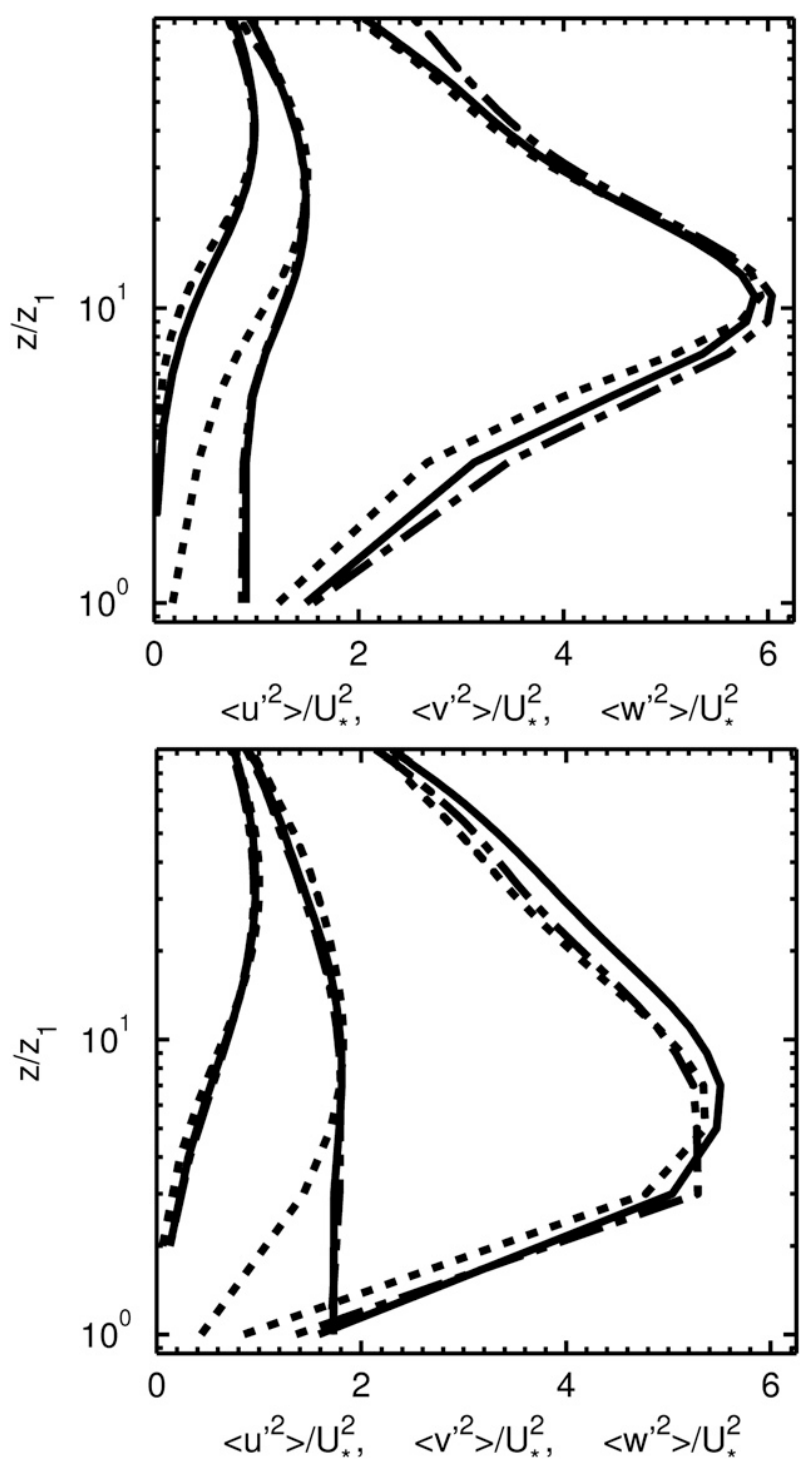

FIG. 6. Vertical profiles of $\left\langle u^{\prime} u^{\prime}\right\rangle$ (largest), $\left\langle v^{\prime} v^{\prime}\right\rangle$ (intermediate), and $\left\langle w^{\prime} w^{\prime}\right\rangle$ (smallest) normalized with $U_{*}^{2}$, for the $3 \mathrm{D}$ roughness model (dotted), highly anisotropic intermittent young sea surface model (solid) and highly anisotropic nonintermittent young sea surface model (dashed-dotted). Results are shown for (top) the SGS TKE-based eddy viscosity SGS model and (bottom) Sullivan's SGS model. The height is normalized by $z_{1}$, which is the height of the tallest breaking waves and where the mean surface wind is matched between the young sea surfaces and the 3D surface.

the $3 \mathrm{D}$ roughness model is used together. Taking account of this overpredicting bias of LES, we will examine the relative difference between the profiles over the $3 \mathrm{D}$ roughness and the young sea surfaces rather than their actual values.

Clearly, the mean vertical gradient near the bottom surface (but above the height of the largest breakers) is reduced over the very young sea surfaces. This gradient
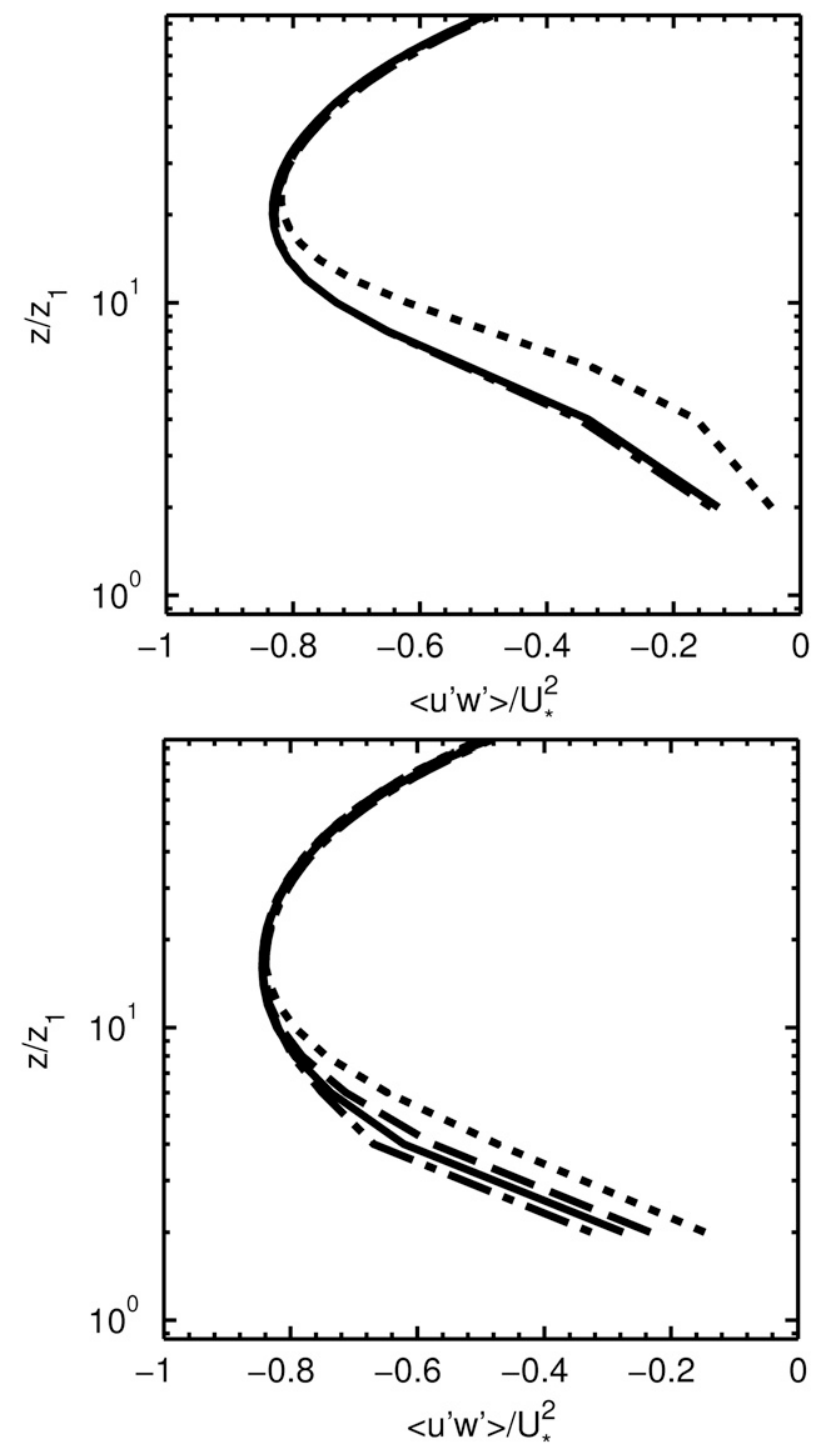

FIG. 7. As in Fig. 6, but for $\left\langle u^{\prime} w^{\prime}\right\rangle$. The dashed line in the bottom panel is the result of the modified simulation discussed in section $4 \mathrm{e}$.

reduction in the roughness sublayer leads to a smaller mean velocity in the logarithmic layer if the wind speed is matched at the height of the largest breakers as in this study. (If we instead match the wind speed and stress in the logarithmic layer, the wind speed at the height of the largest breakers will be larger over the very young sea surfaces.) This finding again implies that the WBL extends significantly higher than the tallest breaking wave height. This is in contrast with previous boundary layer theories (e.g., Kudryavtsev and Makin 2001; Kukulka and Hara 2008a,b; Kukulka et al.2007), in which the surface wave effect is assumed to be contained below the height of the largest breakers. 


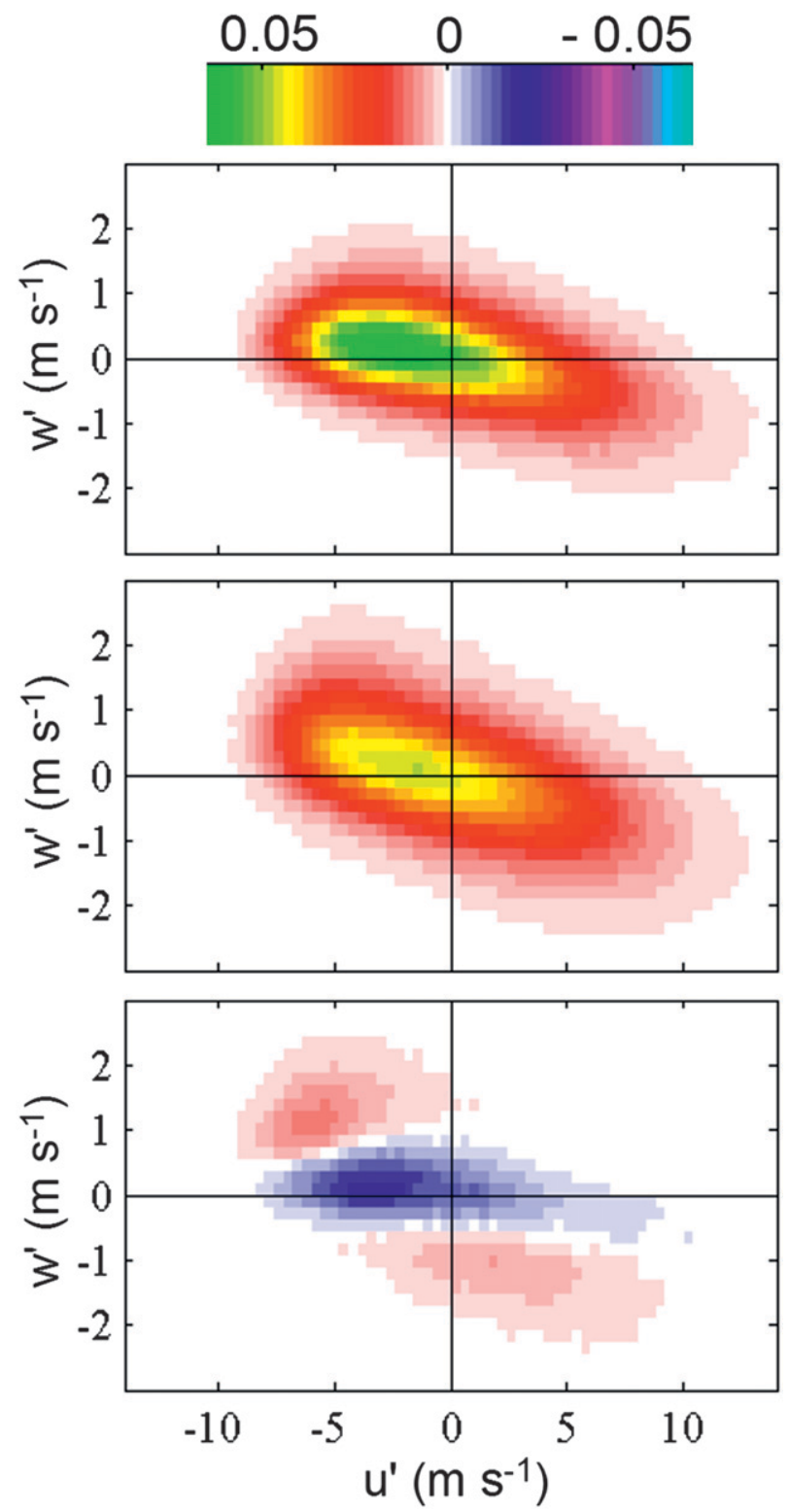

FIG. 8. The joint PDF $\left(\mathrm{s}^{2} \mathrm{~m}^{-2}\right)$ resulting from (top) the 3D roughness model and (middle) the highly anisotropic intermittent young sea surface model at $z=0.31 \mathrm{~m}$ (i.e., the second off-wall $u$ grid level); (bottom) their difference (middle minus top). Sullivan's SGS model is used.

\section{d. Resolution dependence}

Figure 12 compares the results of the high-resolution simulations to the regular-resolution simulations. Their results are very similar except that the near-boundary behaviors occur closer to the bottom boundary for the higher resolution. This is a typical characteristic of LES (e.g., Senocak et al. 2007; Brasseur and Wei 2010).
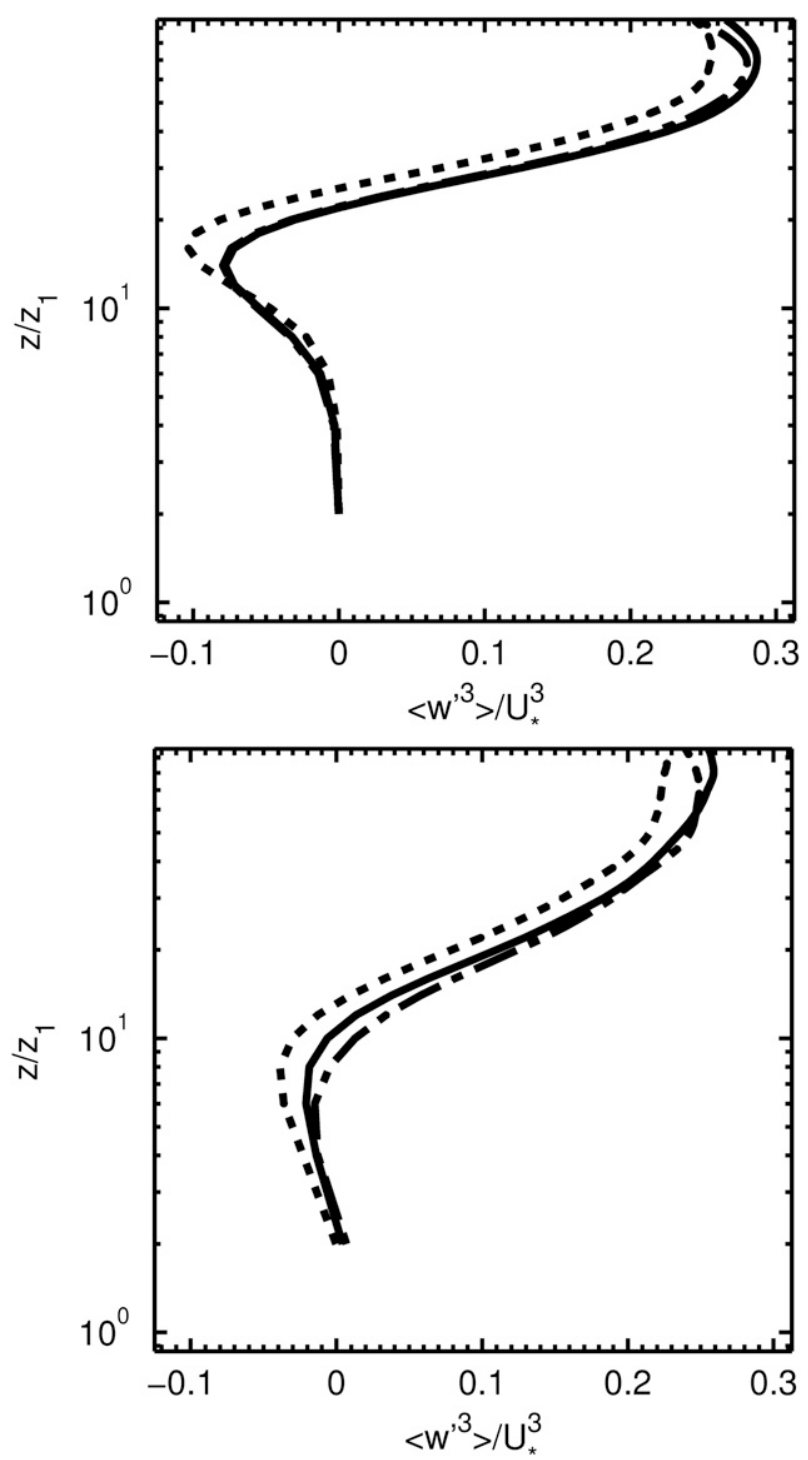

FIG. 9. Vertical profile of $\left\langle\left(w^{\prime}\right)^{3}\right\rangle$ normalized with $U_{*}^{3}$. The normalization of the height is as in previous figures, showing results for (top) the SGS TKE-based eddy viscosity SGS model and (bottom) Sullivan's SGS model, for the 3D roughness model (dotted), highly anisotropic intermittent young sea surface model (solid), and highly anisotropic nonintermittent young sea surface model (dashed-dotted).

\section{e. More realistic shapes and orientations of breaking waves}

For the foregoing, idealized, highly anisotropic sea surface simulations, it was assumed that all breaking crests are perpendicular to the mean near-surface wind, and we set the spanwise form drag to zero. Over real sea surfaces, however, the spanwise form drag does not completely disappear because breaking wave crests have finite lengths and also are not perfectly unidirectional. 

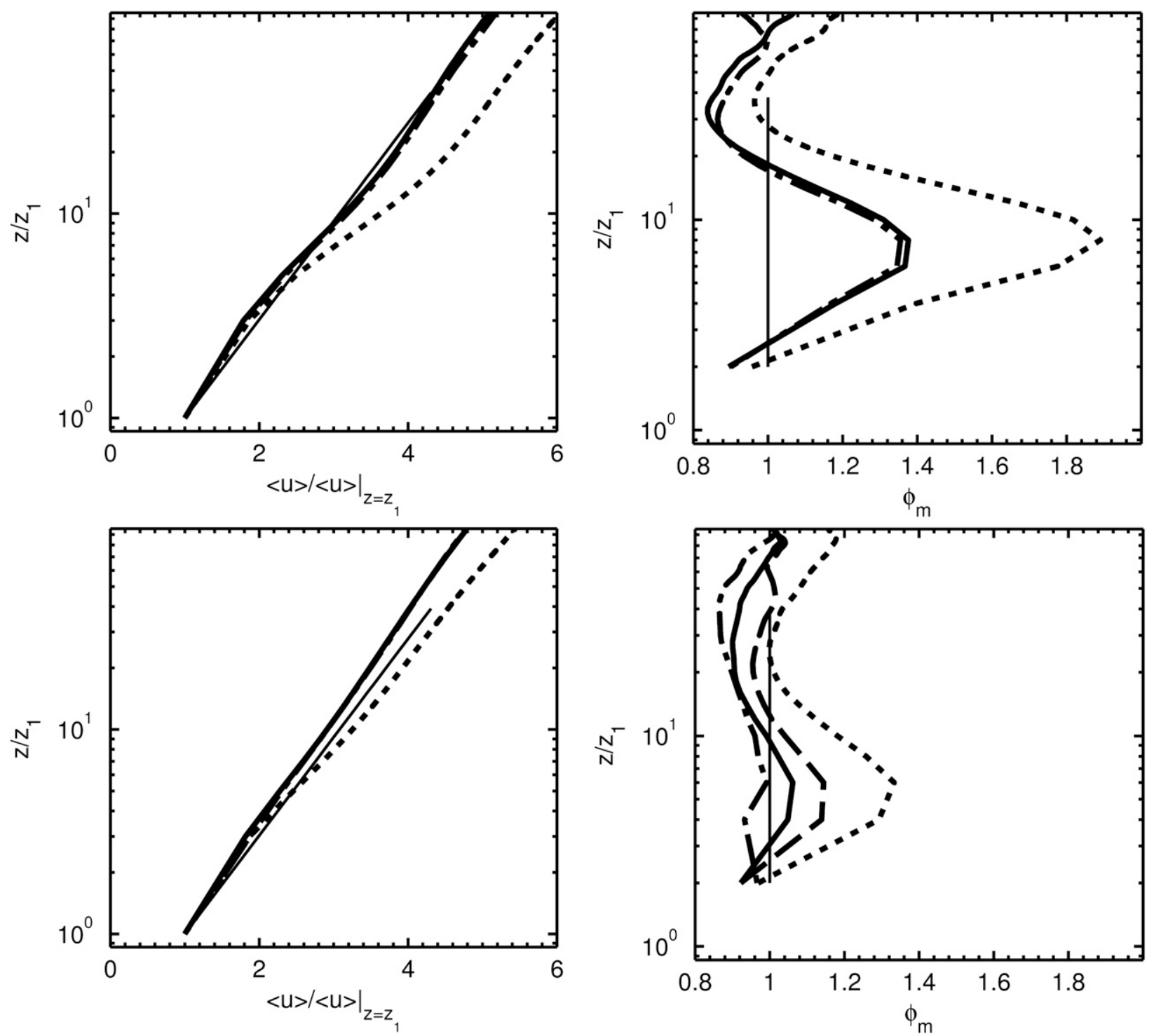

FIG. 10. As in Fig. 9, but for $\langle u\rangle$ normalized with the mean velocity at $z_{1}$. The dashed-dotted line mostly overlaps the solid line. The thin solid line indicates the log profile.

In particular, the orientation of the very small breaking waves can be perpendicular to the local wind rather than the mean wind since they can develop within a very short time scale of the fluctuation of local wind direction.

Although it is difficult to quantify the effect of the finite lengths of breaking crests on the spanwise drag, rough estimation of the spanwise drag due to very small breaking waves is possible. If the small breaking waves are generated in response to the local instantaneous surface wind, then they should appear perpendicular to the local instantaneous surface wind rather than to the mean surface wind. Hence, the form drag over the small breaking waves is opposite to the local wind, being

FIG. 11. Vertical profile of $\phi_{m}$. The normalization of the height is as in previous figures. Results are shown for (top) the SGS-TKEbased eddy viscosity SGS model and (bottom) Sullivan's SGS model, for the 3D roughness model (dotted), highly anisotropic intermittent young sea surface model (solid), and highly anisotropic nonintermittent young sea surface model (dashed-dotted). The thin solid lines is the log profile. The dashed line in the bottom panel is the result of the modified simulation discussed in section $4 \mathrm{e}$.

similar to isotropic roughness. If we choose the cutoff wavenumber $k_{t}$ such that waves at $k>k_{t}$ are generated in response to the local instantaneous surface wind, then Eq. (13) must be modified as

$$
\tau_{y z}(x, y, 0, t)=-\left|\frac{s\left(k_{t}, \infty\right)}{\left.\rho_{a}\langle u\rangle\right|_{z=z_{1}}}\right| v\left(x, y, z_{1}, t\right) .
$$



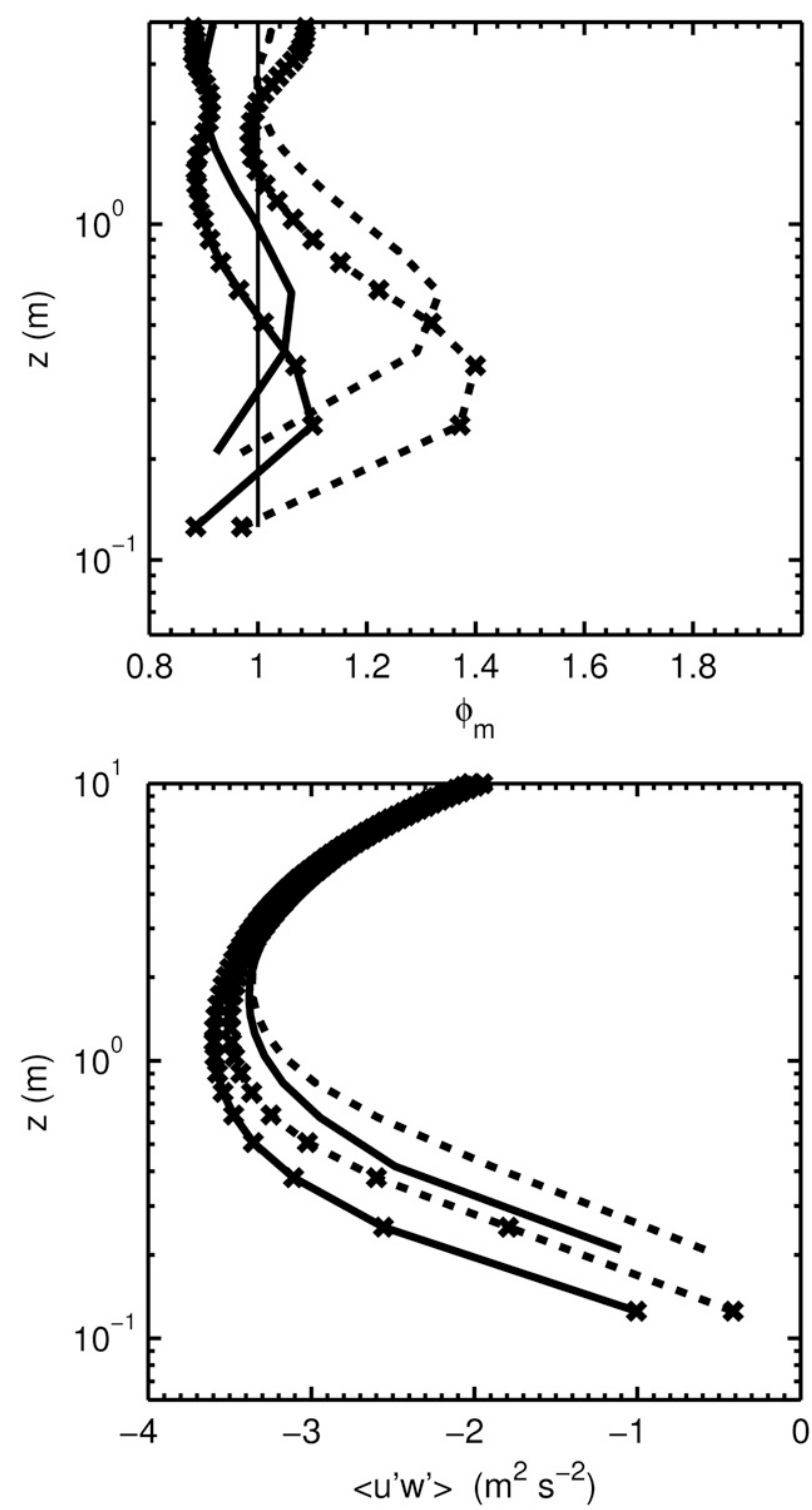

FIG. 12. Resolution dependence for (top) $\phi_{m}$ and (bottom) $\left\langle u^{\prime} w^{\prime}\right\rangle$. Sullivan's SGS model is used; the 3D roughness model (dotted) and highly anisotropic intermittent young sea surface model (solid) are shown. The cross-marked lines are the highresolution simulations, and the lines without crosses are the regular-resolution simulations.

Now, the form drag over the small breaking waves whose wavenumbers are larger than $k_{t}$ is isotropic.

An appropriate value of $k_{t}$ may be found based on the time-dependent amplitudes $a(k, t)$ of wind-driven gravity waves estimated by

$$
a(k, t)=a_{0}(k) \exp \left[\frac{\beta(k)}{2} t\right],
$$

where $a_{0}(k)$ is the initial amplitude and $\beta(k)$ is the (energy) growth rate expressed as

$$
\beta(k)=C_{\beta} \frac{\rho_{a}}{\rho_{w}}\left(\frac{U *}{c}\right)^{2} \omega,
$$

with a constant $C_{\beta}$ (e.g., Belcher and Hunt 1998). Using Eqs. (18) and (19), we can estimate the wavenumbers of those waves that grow fast enough to reach the breaking threshold amplitude $a_{b}=\varepsilon / k$ within the average time scale $t_{b}$ of the simulated fluctuations of local wind direction.

This time scale $t_{b}$ was empirically determined as follows. First, the spatial correlation scale of the fluctuating wind field was determined as $\sim 5 \mathrm{~m}$ from two-point spatial correlations. Next, the advective velocity scale (mean velocity at the lowest grid) was estimated to be $\sim 5 \mathrm{~m} \mathrm{~s}^{-1}$. With these two, the time scale was estimated to be $t_{b} \sim 1 \mathrm{~s}$. The corresponding range of the estimated $k_{t}$ is roughly $15-30 \mathrm{rad} \mathrm{m}^{-1}$ depending on the range of the constant $15 \leq C_{\beta} \leq 30$ and the initial condition $3 \leq a_{b} /$ $a_{0} \leq 10$. Thus, we set $k_{t}=17.0 \mathrm{rad} \mathrm{m}^{-1}$ based on the assumptions $C_{\beta}=25$ (Kukulka and Hara 2008a) and $a_{b} / a_{0}=3$.

Some results of this modified simulation are shown in Figs. 7 and 11. As expected, the results are located between the highly anisotropic surface results and 3D rough surface results. Therefore, the turbulence dynamics highlighted using the more idealized simulations (in particular, enhancement of the QSVs and resulting mixing) likely plays a significant role even over more realistic young sea surfaces.

\section{Conclusions}

For both 3D roughness and very young sea surfaces, the main large-scale turbulence structures near the bottom boundary are wall-attached QSVs. The 3D roughness weakens the swirling motions of these QSVs by strong spanwise form drag. In contrast, the very young sea surfaces exert little spanwise form drag and allow development of more intense, wall-attached QSVs compared to the 3D roughness. The enhanced QSVs result in more intense turbulence and mixing of the fluid. The fluid mixing decreases the vertical velocity shear in the WBL. As a result, the roughness length in the overlying logarithmic layer increases; that is, the mean velocity there decreases if the wind speed and the wind stress are matched at the height of the largest breaking waves. In previous model studies (e.g., Kudryavtsev and Makin 2001; Kukulka et al. 2007), it was assumed that the enhancement of the air-sea momentum flux efficiency (i.e., roughness length or bulk drag coefficient) due to breaking waves was mainly caused by the enhanced streamwise form drag over individual breaking waves. This study, 
however, suggests that the enhancement of the wallattached QSVs due to the anisotropy of the breaking wave geometry may further contribute to the enhanced drag coefficient and the roughness length. The increased wall-attached QSVs enhance strong ejections more than strong sweeps.

These effects are caused by the anisotropy of the dominant horizontal form drag alone, and the intermittency of the breaking waves has only a weak effect of shortening the wall-attached QSVs. Although this study has mainly focused on the idealized cases to highlight the effect of the young sea surfaces, the dynamics found here is likely to play an important role in more realistic young sea states.

Most existing wave boundary layer models assume that the effect of breaking waves are confined below the height of the tallest breaking waves (i.e., the wave boundary layer height is comparable to the largest breaking wave height). This study, however, suggests that the wave boundary layer may extend significantly higher, as much as 10-20 times the breaking wave height.

Acknowledgments. This work was supported by the U.S. National Science Foundation (Grant OCE-0824906). We used the computational resources at National Center for Atmospheric Research (NCAR).

\section{REFERENCES}

Balakumar, B. J., and R. J. Adrian, 2007: Large- and very-largescale motions in channel and boundary-layer flows. Philos. Trans. Roy. Soc., 365A, 665-681.

Belcher, S. E., and J. C. R. Hunt, 1998: Turbulent flow over hills and waves. Annu. Rev. Fluid Mech., 30, 507-538.

Brasseur, J. G., and T. Wei, 2010: Designing large-eddy simulation of the turbulent boundary layer to capture law-of-the-wall scaling. Phys. Fluids, 22, 021303, doi:10.1063/1.3319073.

Choi, H., P. Moin, and J. Kim, 1993: Direct numerical simulation of turbulent flow over riblets. J. Fluid Mech., 255, 503-539.

,-- , and - , 1994: Active turbulence control for drag reduction in wall-bounded flows. J. Fluid Mech., 262, 75-110.

del Álamo, J. C., J. Jiménez, P. Zandonade, and R. D. Moser, 2006: Self-similar vortex clusters in the turbulent logarithmic region. J. Fluid Mech., 561, 329-358.

Dwyer, M. J., E. G. Patton, and R. H. Shaw, 1997: Turbulent kinetic energy budgets from a large-eddy simulation of airflow above and within a forest canopy. Bound.-Layer Meteor., 84, $23-43$.

Flack, K. A., M. P. Schultz, and J. S. Connelly, 2007: Examination of a critical roughness height for outer layer similarity. Phys. Fluids, 19, 095104, doi:10.1063/1.2757708.

Grötzbach, G., 1987: Direct numerical and large eddy simulations of turbulent channel flows. Encyclopedia of Fluid Mechanics, N. P. Cheremisinoff, Ed., Gulf Publishing Co., 1337-1391.

Ikeda, T., and P. A. Durbin, 2007: Direct simulations of a roughwall channel flow. J. Fluid Mech., 571, 235-263.

Karniadakis, G., and K.-S. Choi, 2003: Mechanisms on transverse motions in turbulent wall flows. Annu. Rev. Fluid Mech., 35, $45-62$.
Kim, K., R. J. Adrian, S. Balachandar, and R. Sureshkumar, 2008: Dynamics of hairpin vortices and polymer-induced turbulent drag reduction. Phys. Rev. Lett., 100, 134504, doi:10.1103/ PhysRevLett.100.134504.

Kudryavtsev, V. N., and V. K. Makin, 2001: The impact of air-flow separation on the drag of the sea surface. Bound.-Layer Meteor., 98, 155-171.

Kukulka, T., and T. Hara, 2008a: The effect of breaking waves on a coupled model of wind and ocean surface waves. Part I: Mature seas. J. Phys. Oceanogr., 38, 2145-2163.

$\ldots$, and $-2008 \mathrm{~b}$ : The effect of breaking waves on a coupled model of wind and ocean surface waves. Part II: Growing seas. J. Phys. Oceanogr., 38, 2164-2184.

,-- , and S. E. Belcher, 2007: A model of the air-sea momentum flux and breaking-wave distribution for strongly forced wind waves. J. Phys. Oceanogr., 37, 1811-1828.

Lee, J. H., S.-H. Lee, K. Kim, and H. J. Sung, 2009: Structure of the turbulent boundary layer over a rod-roughened wall. Int. J. Heat Fluid Flow, 30, 1087-1098.

Melville, W. K., F. Veron, and C. J. White, 2002: The velocity field under breaking waves: Coherent structures and turbulence. J. Fluid Mech., 454, 203-233.

Min, T., and J. Kim, 2004: Effects of hydrophobic surface on skinfriction drag. Phys. Fluids, 16, 55-58.

Moeng, C.-H., 1984: A large-eddy-simulation model for the study of planetary boundary-layer turbulence. J. Atmos. Sci., 41, 2052-2062.

, and P. P. Sullivan, 1994: A comparison of shear- and buoyancy-driven planetary boundary layer flows. J. Atmos. Sci., 51, 999-1022.

Morris, S. C., S. R. Stolpa, P. E. Slaboch, and J. C. Klewicki, 2007: Near-surface particle image velocimetry measurements in a transitionally rough-wall atmospheric boundary layer. J. Fluid Mech., 580, 319-338.

Phillips, O., 1985: Spectral and statistical properties of the equilibrium range in wind-generated gravity waves. J. Fluid Mech., 156, 505-531.

Piomelli, U., 2008: Wall-layer models for large-eddy simulations. Prog. Aerosp. Sci., 44, 437-446.

Pope, S. B., 2000: Turbulent Flows. Cambridge University Press, $771 \mathrm{pp}$.

Senocak, I., A. S. Ackerman, M. P. Kirkpatrick, D. E. Stevens, and N. N. Mansour, 2007: Study of near-surface models for largeeddy simulations of a neutrally stratified atmospheric boundary layer. Bound.-Layer Meteor., 124, 405-424.

Sjöblom, A., and A.-S. Smedman, 2003: Vertical structure in the marine atmospheric boundary layer and its implication for the inertial dissipation method. Bound.-Layer Meteor., 109, $1-25$.

Stoll, R., and F. Porté-Agel, 2006: Effect of roughness on surface boundary conditions for large-eddy simulation. Bound.-Layer Meteor., 118, 169-187.

Sullivan, P. P., and J. C. McWilliams, 2010: Dynamics of winds and currents coupled to surface waves. Annu. Rev. Fluid Mech., 42, $19-42$.

_, _ and C.-H. Moeng, 1994: A subgrid-scale model for large-eddy simulation of planetary boundary-layer flows. Bound.-Layer Meteor., 71, 247-276.

,$- \ldots$, and $—$, 2000: Simulation of turbulent flow over idealized water waves. J. Fluid Mech., 404, 47-85.

,$- \ldots$, and W. K. Melville, 2004: The oceanic boundary layer driven by wave breaking with stochastic variability. Part 1 : Direct numerical simulations. J. Fluid Mech., 507, 143-174. 
_ J. B. Edson, T. Hristov, and J. C. McWilliams, 2008: Largeeddy simulations and observations of atmospheric marine boundary layers above nonequilibrium surface waves. $J$. Atmos. Sci., 65, 1225-1245.

Tomkins, C. D., and R. J. Adrian, 2003: Spanwise structure and scale growth in turbulent boundary layers. J. Fluid Mech., 490, 37-74.

- and _- 2005: Energetic spanwise modes in the logarithmic layer of a turbulent boundary layer. J. Fluid Mech., 545, 141-162.
Veron, F., G. Saxena, and S. K. Misra, 2007: Measurements of the viscous tangential stress in the airflow above wind waves. Geophys. Res. Lett., 34, L19603, doi:10.1029/2007GL031242.

Volino, R. J., M. P. Schultz, and K. A. Flack, 2009: Turbulence structure in a boundary layer with two-dimensional roughness. J. Fluid Mech., 635, 75-101.

Wyngaard, J. C., L. J. Peltier, and S. Khanna, 1998: LES in the surface layer: Surface fluxes, scaling, and SGS modeling. J. Atmos. Sci., 55, 1733-1754. 